\title{
Arrhythmia Classification Using Radial Basis Function Network With Selective Features From Empirical Mode Decomposition
}

\author{
Saumendra Kumar Mohapatra, ITER, Siksha 'O’ Anusandhan (Deemed), India \\ (iD) https://orcid.org/0000-0002-5167-5695
}

Mihir Narayan Mohanty, ITER, Siksha 'O’ Anusandhan (Deemed), India

\begin{abstract}
In this piece of work, the authors have attempted to classify four types of long duration arrhythmia electrocardiograms (ECG) using radial basis function network (RBFN). The data is taken from Massachusetts Institute of Technology-Beth Israel Hospital (MIT-BIH) arrhythmia database, and features are extracted using empirical mode decomposition (EMD) technique. For most informative contents average power (AP) and coefficient of dispersion (CD) are evaluated from six intrinsic mode function (IMFs) of EMD. Principal component analysis (PCA) is used for feature reduction for effective classification using RBFN. The performance is shown in the result section, and it is found that the classification accuracy is $95.98 \%$.
\end{abstract}

\section{KEYWORDS}

Arrhythmia Classification, Cardiac Disease, ECG, EMD, IMF, Neural Network, PCA, RBFN

\section{INTRODUCTION}

Electrocardiogram (ECG) is one of the important sources for analysing any kind of cardiac disease. Electrical impulses that occur during each heartbeat can be visualized in ECG. Accurate analysis of the ECG is most important in the diagnosis of heart disease. Any changes in a normal ECG can occur during various cardiac abnormalities like atrial fibrillation, ventricular tachycardia, myocardial infarction, hypokalemia, etc. Arrhythmia is a type of cardiac illness and it can be detected by strongly analysing waves in ECG (Wu \& Rangayyan, 2009). It causes due to the abnormal electrical activity in the heart. In this case the heartbeat rhythms either fast or slow. The factors that cause cardiac arrhythmia are smoking and alcohol consumption, mental stress, diet, diabetes, etc.

Due to the manual data analysis system, it is difficult to extract useful information from the clinical data. Computer-based automated disease diagnosis system will be most useful in medical sectors. The system will automatically take the decision by analyzing the data collected through

This article, published as an Open Access article on November 6, 2020 in the gold Open Access journal, International Journal of Cognitive Informatics and Natural Intelligence (converted to gold Open Access January 1, 2021), is distributed under the terms of the Creative Commons Attribution License (http://creativecommons.org/licenses/by/4.0/) which permits unrestricted use, distribution, and production in any medium, provided the author of the original work and original publica- 
different medical tests. Data include ECG, Ultrasonic images, Magnetic resonance imaging (MRI) are useful for analysis and diagnosis. Machine learning-based medical data analysis is one of the advanced technology that can reduce human interaction by enhancing machine efficiency and will be cost-effective. For early detection of cardiac arrhythmia, a real-time automatic ECG analysis system will be the best support to the physicians (Polat \& Güneş, 2007). The characteristic of the ECG varies for different patients in different physical and temporal conditions. Due to these variations in ECG, the task is very difficult for analysis. This requires to develop an automatic ECG classification system (Hoekema, Uijen, \& Van Oosterom, 2001). For the development of accurate ECG classifier it is important to extract useful features from data. Several methods for the analysis and classification of ECG signals have been proposed by the researchers over the past few decades. Wavelet Transform (WT) (Tantawi, Revett, Salem, \& Tolba, 2015), frequency analysis (Lin, 2008), statistical techniques (Willems \& Lesaffre, 1987), filter banks (Afonso, Tompkins, Nguyen, \& Luo, 1999), support vector machines (Alonso-Atienza, Morgado, Fernandez-Martinez, García-Alberola, \& Rojo-Alvarez, 2013), artificial neural network (Hu, Tompkins, Urrusti, \& Afonso, 1993) are the most used methods for ECG analysis and classification.

Due to the non-stationary nature of the ECG signal, EMD is considered in the proposed work for decomposition and feature extraction. Total six number of Intrinsic mode functions (IMFs) are considered as the basic feature. In order to extract useful feature, average power (AP) and coefficient of dispersion (CD) are found from these six IMFs. AP is the mean by which the signal is characterized for reusing important information of the signal. $C D$ of a signal signifies variation of coefficients. Here in the proposed work CD is used for building a feature vector by calculating the probability distribution of the IMFs. Again the dimension of the feature vector is reduced by using principal component analysis (PCA) method. Radial basis function network (RBFN) is used for classifying these selected ECG features. Massachusetts institute of Technology-Beth Israel hospital (MIT-BIH) arrhythmia data has been used for classifying four major types of arrhythmia. A few approaches have been considered earlier for large scale Radial Basis Function Network. In this case the data may be large scale along with the higher variance value. However, in this case, the variance is considered as 2 .

\section{RELATED LITERATURE}

Automated ECG classification can help the cardiologist for the diagnosis of any type of cardiac abnormalities. In the last few decades, several algorithms have been developed by the researchers for the automatic classification of the cardiac signal. Preprocessing, feature extraction, and classification are the three basic steps in ECG signal classification and multiple methods have been applied by the researchers for each of these processes.

Classification of normal and coronary artery disease was performed by applying higher-order statistics and spectra (HOS) method (Acharya et al., 2017). From each heartbeat, HOS bispectrum and cumulants features were extracted. For dimensionality reduction, authors have taken PCA. Knearest neighbor (KNN) and decision tree classifiers were used for the classification purpose as the performance of these classifiers with fewer features are better. Support vector machine (SVM) classifier was used by the authors for the detection of coronary artery disease (Babaoğlu, Fındık, \& Bayrak, 2010). They have used PCA for the feature selection purpose and $79 \%$ classification accuracy was achieved by them. Genetic algorithm (GA) and binary particle swarm optimization (BPSO) methods were used for feature selection (Babaoglu, Findik, \& Ülker, 2010). SVM with $k$-fold cross-validation was considered for classification purpose and $81 \%$ classification accuracy was obtained from their proposed system. S-transform and wavelet transform was used for feature extraction (Das \& Ari, 2014). Multi-layer perceptron was considered for the classification of normal and abnormal ECG beats. A long term ECG classification framework was presented in a study (Kiranyaz, Ince, Pulkkinen, $\&$ Gabbouj, 2011). Exhaustive $k$-means clustering technique was used for getting an optimal number of key beats and master beats from the ECG waveform. Backpropagation algorithm based classifier 
was introduced by the authors for the classification purpose. Average accuracy for their proposed system was $99.04 \%$ and it was quite good. But they have only considered the representative beats for the arrhythmia classification purpose and the computational time in their work is more. In certain cases, multiple classifiers for ECG classification were used. Automatic detection of the coronary artery from the cardiac signal using four different classifiers was performed (Giri et al., 2013). In the first stage, authors have applied Discrete Wavelet Transform (DWT) for decomposition purpose. Further, the dimensions of the wavelet coefficients were reduced by applying PCA, Linear discriminant analysis (LDA), and Independent component analysis (ICA). After the selection of suitable features from the cardiac signal, the classification was performed by using KNN, SVM, Probabilistic neural network (PNN), and Gaussian mixture model (GMM). From their result, it has been observed that ICA with GMM classifier was giving better result as compare to the other three types of classifiers.

Soft computing techniques have been utilized for ECG signal classification in different studies. Radial basis function neural network (Lewenstein, 2001) and Block-based neural network (BBNN) (Jiang \& Kong, 2007) was applied in some studies for automatic ECG classification. Six types of ECG was classified by using particle swarm optimization (PSO) and RBFN in (Korürek \& Doğan, 2010) and they have obtained better classification accuracy in their proposed work. Input for their proposed BBNN was Hermite transform coefficients and the time interval between two adjacent R-peaks (amplitude of $\mathrm{R}$ wave is maximum) of each heartbeat. They have conducted their simulation by using MIT-BIH arrhythmia data set. Classification of six types of ECG was done by using a probabilistic neural network (PNN) (Yu \& Chen, 2007). Discrete wavelet transform was first applied for the decomposition and feature extraction of the cardiac signal. PNN classifier was used for classifying the feature sets and $99 \%$ of accuracy was obtained as they claimed. However, it was not found in case of long term ECG because they have only considered only eleven features for their classifier. Similarly, the neural network was used to predict arrhythmia (Abdou, Ngom, \& Niang, 2018). They have claimed that the classification accuracy was $67 \%$. Also, PNN was used for classification of eight types of heartbeats in (Gutiérrez-Gnecchi et al., 2017) where they have reported $92.75 \%$ classification accuracy. Classification of ECG using multilayer perceptron (MLP) was conducted by the researchers in (Güler $\&$ Übeyl1, 2005). Four types of ECG beats were classified in their work and they have taken different types of mother wavelets for validating their result. In Daubechies2 (db2) wavelet type, the authors have obtained a better result as compare to other wavelet types. Combination of DWT and ANN was proposed by the authors in (Prasad \& Sahambi, 2003). They have verified their work with noisy and clean ECG signal. ECG without power line interference and baseline wander was performing best as compare to noisy. Data mining method such as attribute selection and expectation maximization(EM) based data clustering was introduced by authors in (Sufi \& Khalil, 2010). The dimension of the feature vector was reduced by correlation-based selection technique. A rule-based classifier was designed for classifying the ECG and Higher-order spectra (HOS) was introduced by the authors in (Martis et al., 2013). For reduction of the dimension, Independent component analysis (ICA) was considered and the classification was occurred by using multiple classifiers. From their result, KNN classifier was giving better classification accuracy as compared to other classifiers. Optimum path forest (OPF) based classifier was chosen by the authors in (de Albuquerque et al., 2018). In their work, they have compared their result for six types of feature extraction technique, and three types of classification technique. All those techniques were applied in the same MIT-BIH database.

However, from the literature, it can be observed that researchers have not particularly focussed on Arrhythmia dataset for long duration properly. Also the suitable of features were not well deduced. As the effective machine learning technique RBFN has chosen in this work. This work proposes with suitable features extracted by EMD to make the system easy operation and applicable for medical data mining with minimum number of features. With less number of features RBFN exhibits better result (Palo, Mohanty, \& Chandra, 2016) 


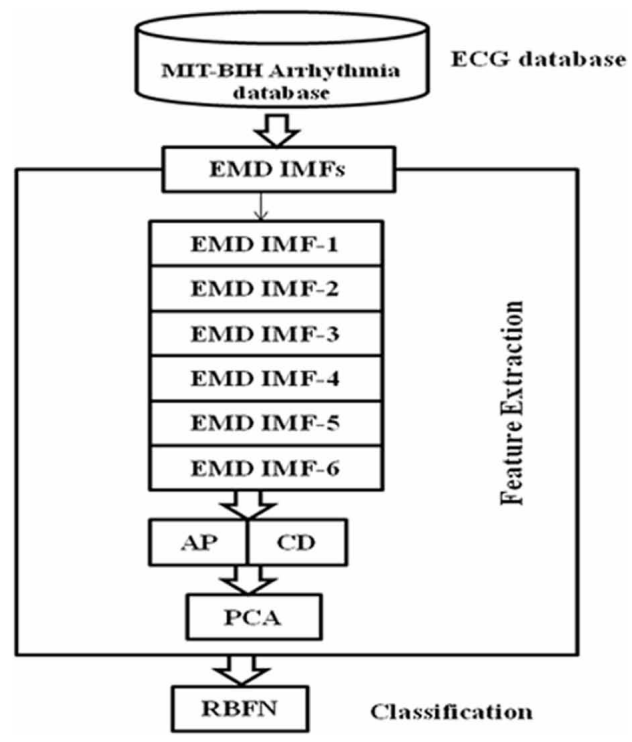

\section{PROPOSED METHODOLOGY}

Most of the medical dataset are dispersed, widespread, and assorted. However, data mining is a robust technique for extracting invisible, predictive and actionable information from the extensive databases. This technique is an exciting field of machine learning and capable of solving various kinds of real-world problems. It is interesting to discover hidden patterns and valuable knowledge from a large dataset. However, the health care industry is another field where a substantial amount of data are collected using different clinical reports and patient manifestations. The proposed work provides an automatic ECG classification system and is based on EMD and RBFN approach. In Figure 1, the proposed framework for automatic ECG, classification is presented.

\subsection{Data Collection}

ECG data is collected from MIT-BIH-Arrhythmia repository (Moody \& Mark, 2001). This database contains 48 number of two-channel ECG data with $360 \mathrm{~Hz}$ sampling frequency. The signals have been recorded at Boston's Berth Israel Hospital (BIH). Total four types of ECG signal such as Normal, Left Bundle Branch Block (LBBB), Atrial premature beats (APB), and Right Bundle Branch Block $(\mathrm{RBBB})$ are available in this database. After collecting the ECG data from this open source database, the feature extraction and classification is done in the next step.

\subsection{Empirical Mode Decomposition}

Empirical Mode Decomposition (EMD) is a suitable technique to decompose any signal. Exclusively it is most useful for non-stationary signal decomposition. The signal is decomposed into a number of intrinsic mode functions (IMFs) and residual by shifting process (Huang et al., 1998; Wang, Chen, Qiao, Wu, \& Huang, 2010). Some predefined functions are required for representing the signal in traditional data analysis methods like Fourier Transform and Wavelet Transform. But EMD relies on a fully data-driven approach and does not require any predefined function (Blanco-Velasco, Weng, \& Barner, 2008). Steps involved for EMD is described as follows:

Step- I: Consider the signal $s(t)$ and determine both maxima and minima. 
Step-II: Find out the upper $e_{u}(t)$ and lower $e_{l}(t)$ envelopes of the signal $s(t)$ using the cubic spline interpolation technique (Damerval, Meignen, \& Perrier, 2005).

Step-III: Find out the envelope mean $e_{m}(t)=\frac{e_{u}(t)+e_{l}(t)}{2}$.

Step-IV: Extract the detail $d_{1}(t)=s(t)-e_{m}(t)$.

Step- V: If the mean of $d_{1}(t)=0$ and the number of extrema is equal or differ by at most one from the zero-crossing then $d_{1}(t)$ is an IMF otherwise repeat step I until satisfying the condition.

Step- VI: Find out the residue $r_{1}(t)=s(t)-d_{1}(t)$. Last residual will be considered as the trend.

After obtaining all the IMFs from the input signal $s(t)$, again it is reconstructed by:

$s(t)=\sum_{i=0}^{n} d_{i}(t)+r_{n}(t)$

where $n$ is the number of IMFs and it is orthogonal to each other (Sahani \& Dash, 2018).

Average power (AP) of the first six IMFs is calculated for summarizing the information of the signal. This can be used as the feature vector for classification. In the proposed work AP is calculated by:

$A P=\frac{I M F_{l}^{2}(1)+I M F_{l}^{2}(2)+I M F_{l}^{2}(3)+I M F_{l}^{2}(4)+\ldots \ldots+I M F_{l}^{2}(N)}{N}$

where $l=1,2,3,4,5,6$ and $N$ is the length of each IMF.

Again the coefficient of dispersion (CD) is calculated and it measures the relative variation of a signal. Mostly it is used to measure the variability in two or more series. CD of first six IMFs is calculated by relative dispersion (Abdalla et al., 2019). The CD feature vector is obtained by equation 5 :

$\mu=\frac{1}{N} \sum_{n=1}^{N} I M F_{l}(n)$

$\sigma=\left[\frac{1}{N} \sum_{n=1}^{N} I M F_{l}(n)-\mu^{2}\right]^{1 / 2}$

$C D=(\sigma / \mu)^{2}$

\subsection{Principal Component Analysis (PCA)}

Sometime the classifier's performance may affect due to the high dimension feature set. In dimensionality reduction technique the data encoding approach is applied for obtaining a reduced and compressed data set. PCA is a statistical dimension reduction technique in which the redundant 
information from the dataset is removed for decreasing the number of the attribute. It converts the observed correlated variables into linearly uncorrelated variables. These set of values are considered as principal components. It works in such a way that the first principal component has the largest possible variance and each subsiding component has the highest possible to match with large scale RBFN model in this case. It is sensitive to the relative scaling of the original variables. (Moreira, de Leon Ferreira, \& Horváth, 2019). Steps involved in finding dimensionally reduced feature vector using PCA is discussed in the following steps:

\section{Step 1: Standardization}

The aim of this step is to standardize the range of the continuous initial variables so that each one of them contributes equally to the analysis. More specifically, the reason why it is critical to perform standardization prior to PCA, is that the latter is quite sensitive regarding the variances of the initial variables. That is if there are large differences between the ranges of initial variables, those variables with larger ranges will dominate over those with small which will lead to biased results. So, transforming the data to comparable scales can prevent this problem. Mathematically it can be done by subtracting the mean $m$ from the value of each variable $v$ then dividing the result by the standard deviation $d$ :

$$
S=\frac{v-m}{d}
$$

After completing the standardization all the variables will be transferred to the same scale.

\section{Step 2: Covariance Matrix computation}

The aim of this step is to understand how the variables of the input data set are varying from the mean with respect to each other, or in other words, to see if there is any relationship between them. Because sometimes, variables are highly correlated in such a way that they contain redundant information. So, in order to identify these correlations, we compute the covariance matrix.

Step 3: Compute the eigenvectors and eigenvalues of the covariance matrix to identify the principal components

Eigenvectors and eigenvalues are the linear algebra concepts that we need to compute from the covariance matrix in order to determine the principal components of the data. Principal components are new variables that are constructed as linear combinations or mixtures of the initial variables. These combinations are done in such a way that the new variables (i.e., principal components) are uncorrelated and most of the information within the initial variables is squeezed or compressed into the first components.

\section{Step 4: Feature vector}

As it is derived in the previous step, computing the eigenvectors and ordering them by their eigenvalues in descending order, then find the principal components in term of significance. In this step, it is decided that whether all these components are kept or discarding those elements that have less significance (of low eigenvalues), and form with the remaining ones a matrix of feature vectors. So, the feature vector is simply a matrix that has as columns the eigenvectors of the components that 
has been decided to keep. This makes it the first step towards dimensionality reduction because if we choose to keep only $p$ eigenvectors (components) out of $n$, the final data set will have only $p$ dimensions.

Step 5: Recast the data along the axes of the principal component

In this step, which is the last one, the aim is to use the feature vector formed using the eigenvectors of the covariance matrix, to reorient the data from the original axes to the ones represented by the principal components (hence the name Principal Components Analysis). This is done by multiplying the transpose of the original data set by the transpose of the feature vector.

The features are selected as described and used for classification. The classification technique is explained in the following section.

\subsection{Radial Basis Function Network}

Radial basis function network (RBFN) consists of an input layer, a hidden layer and a linear output layer as presented in Figure 2. In the proposed RBFN total of 10 numbers of input, 7 hidden, and 4 output neurons are considered. The input neuron size is same as the number of features. The classifier classifies total four types of ECG class. So the output neuron size is 4 . Total 7 hidden neurons are there in hidden layer because if we use more number of neurons then there is a chance of overfitting. Also by using too few neurons in hidden layer will create the underfitting problem. Overfitting and underfitting will affect the performance of the classifier (Hagan, Demuth, \& Beale, 1997). In this case, the Gaussian kernel is used as a kernel function. The hidden layer depends on a non-linear RBF kernel function (Phooi \& Ang, 2006; Ram \& Mohanty, 2019). The output of the network is found as the distance between the input vector and the vector of the center of the Gaussian function and can be expressed as:

$$
\left[\begin{array}{c}
y 1 \\
y 2 \\
\vdots \\
y_{j}
\end{array}\right]=\left[\begin{array}{ccccc}
R\left\|x_{1}-c_{1}\right\| & R\left\|x_{1}-c_{2}\right\| & \cdots & R\left\|x_{1}-c_{j}\right\| \| \\
R\left\|x_{2}-c_{1}\right\| & R\left\|x_{2}-c_{2}\right\| & \cdots & R\left\|x_{2}-c_{j}\right\| \\
\vdots & \vdots & \vdots & \vdots \\
R\left\|x_{j}-c_{1}\right\| & R\left\|x_{j}-c_{2}\right\| & \cdots & R\left\|x_{j}-c_{j}\right\| \|
\end{array} \|\left[\begin{array}{c}
w_{1} \\
w_{2} \\
\vdots \\
w_{j}
\end{array}\right]\right.
$$

where $R$ is the RBF, $c_{j}$ is the center, $\left\|x-c_{j}\right\|$ is the distance between input and the center. $x_{1}, x_{2}, \ldots x_{j}$ are the input neurons, $y_{1}, y_{2} \ldots y_{j}$ are the output neurons and $w_{1}, w_{2}, \ldots w_{j}$ are the weights of the network. The target output is obtained by updating the corresponding weights. The output to weight and input is given as:

\section{Figure 2. Structure of RBF Network}

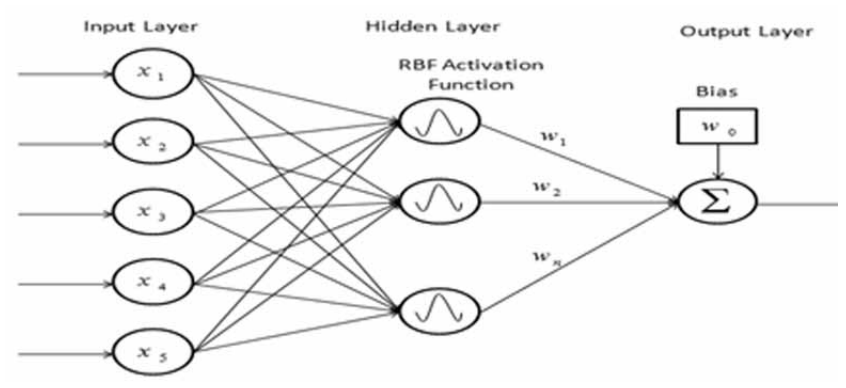




$$
y=\sum_{j=1}^{N} R\left(\left\|x-c_{j}\right\|\right) w_{j}
$$

where $w_{j}$ is the weight of the $j^{\text {th }}$ center and $N$ is the length of the data. The network is operated with the kernel function that is Gaussian and is expressed as:

$$
R\left(\left\|x-c_{j}\right\|\right)=\exp \left[-\frac{\left(x-c_{j}\right)^{2}}{2 \sigma^{2}}\right]
$$

where $\sigma$ is the width of the center.

\section{RESULTS}

Four types of ECG has been considered in this work for classification. The data is collected from the MIT-BIH database and corresponding signals are shown in Figure 3 through Figure 6. After successive decomposition using EMD, IMF and residual for four types of ECG are presented in Figure 7 through 10. First six IMFs are considered for calculation of AP, and CD. The feature vector of size 12 that obtained from AP and CD is subjected to PCA. It reduces the feature vector size to 10 and this new feature vector is considered for classification using RBFN.

Figure 3. Normal ECG signal

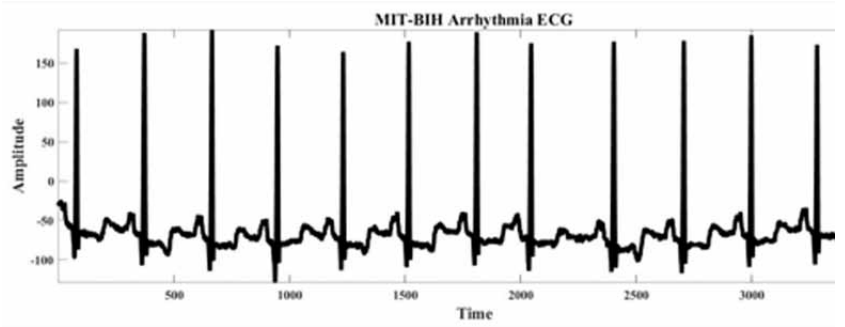

Figure 4. LBBB ECG

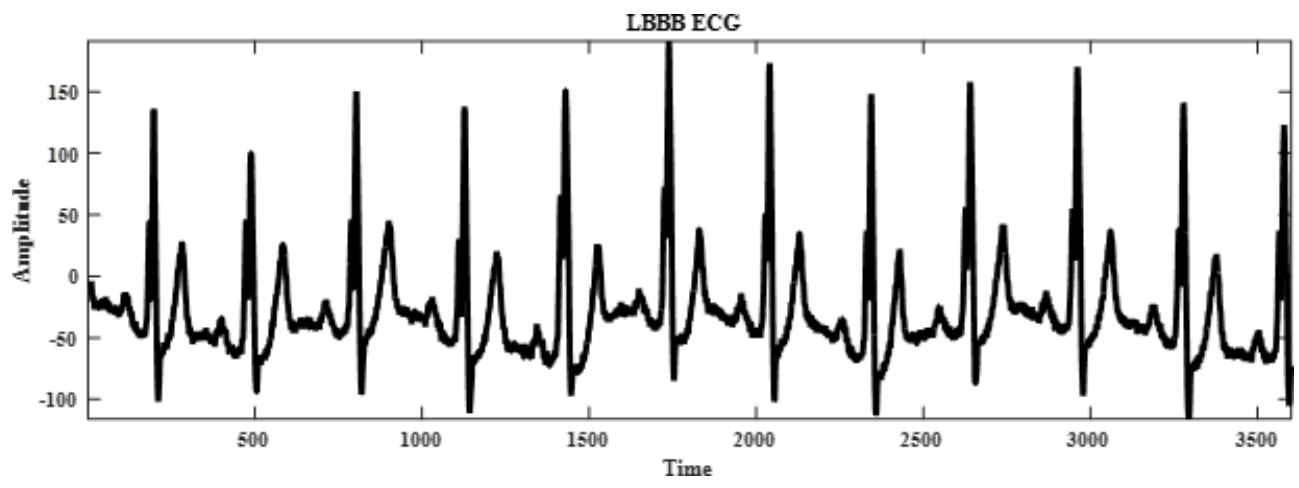


Figure 5. APB ECG

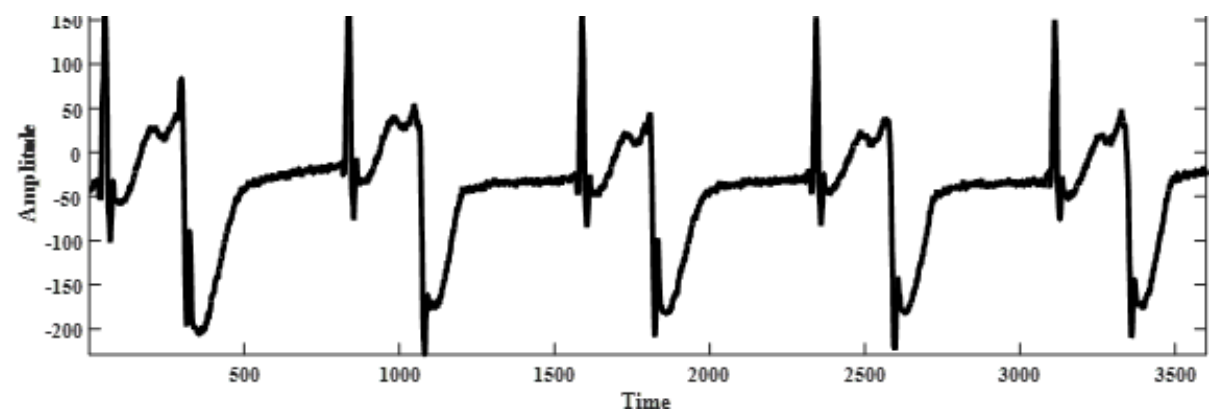

Figure 6. RBBB ECG

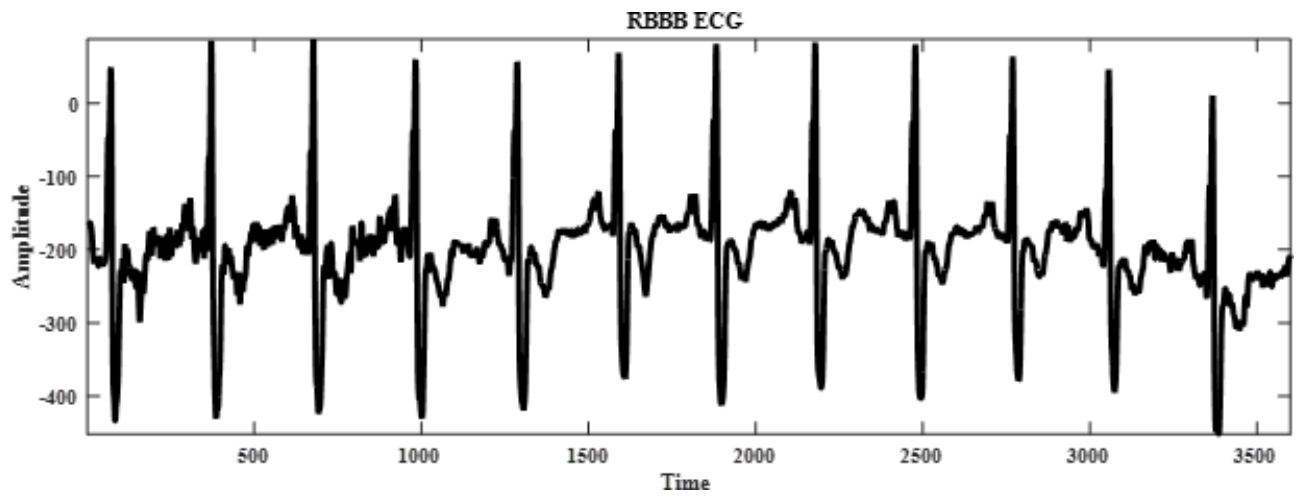

Figure 7. IMFs and residual of normal ECG

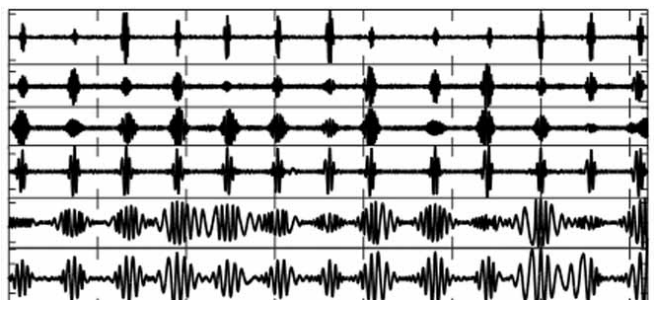

Figure 8. IMFs and residual of LBBB ECG

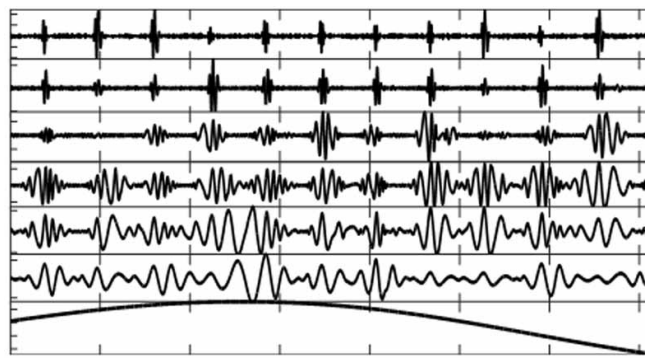


Figure 9. IMFs and residual of APB ECG

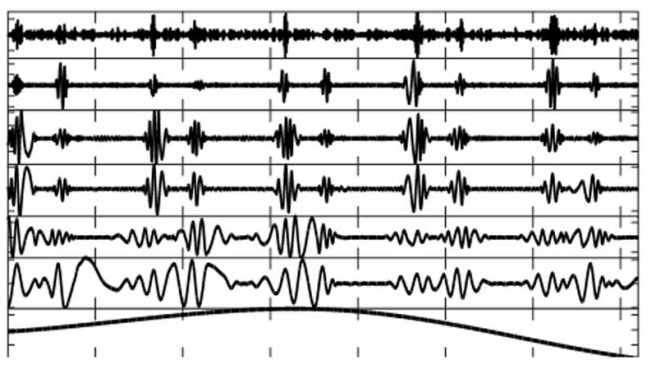

Figure 10. IMFs and residual of RBBB ECG

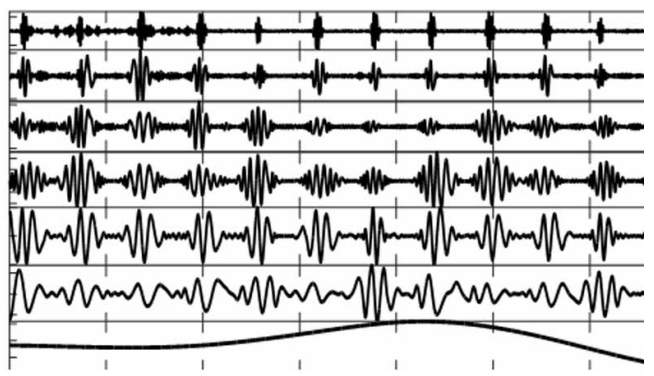

In the normal ECG plot, it can be noticed that the shape of all waves is smooth and not notched and peaked. But in LBBB ECG the QRS duration is more than 120 millisecond. In APB ECG the P wave generally looks abnormal and the QRS complex looks narrow. In RBBB ECG the QRS pattern appears intermittently.

About $80 \%$ of the ECG data is considered as the training set and rest $20 \%$ data is considered as the validation dataset. Performance of the classifier is firstly measured by the confusion matrix as presented in Table 1.

Based on the obtained confusion matrix the performance is measured by calculating sensitivity, specificity, and accuracy for each class of arrhythmia. These performance measuring parameters are calculated by:

$$
\text { sensit. }=\frac{\operatorname{Tr} P}{\operatorname{Tr} P+F l N}
$$

spec. $=\frac{\operatorname{Tr} N}{\operatorname{Tr} N+F l P}$

$$
A c c .=\frac{\operatorname{Tr} P+\operatorname{Tr} N}{N} * 100
$$


Table 1. Confusion matrix of RBFN classifier

\begin{tabular}{|l|l|l|l|l|}
\hline \multicolumn{1}{|c|}{ Beat Type } & \multicolumn{1}{c|}{ Normal } & \multicolumn{1}{c|}{ LBBB } & \multicolumn{1}{c|}{ APB } & \multicolumn{1}{c|}{ RBBB } \\
\hline Normal & 495 & 5 & 8 & 0 \\
\hline LBBB & 0 & 66 & 2 & 0 \\
\hline APB & 40 & 8 & 200 & 2 \\
\hline RBBB & 1 & 1 & 1 & 24 \\
\hline
\end{tabular}

Here $\operatorname{Tr} P=$ True positive and $\operatorname{Tr} N=$ True Negative, $F l P=$ False positive, $F l N=$ False negative, $\mathrm{N}=$ number of samples. All these performance measuring parameters for the proposed classifier are presented in Table 2 .

Table 2. Classification result of RBFN classifier

\begin{tabular}{|l|l|l|l|}
\hline \multicolumn{1}{|c|}{ Class } & \multicolumn{1}{|c|}{ Sensitivity (\%) } & \multicolumn{1}{c|}{ Specificity (\%) } & \multicolumn{1}{c|}{ Accuracy (\%) } \\
\hline Normal & 97.44 & 88.08 & 93.55 \\
\hline LBBB & 97.05 & 98.12 & 98.12 \\
\hline APB & 80 & 98.17 & 92.84 \\
\hline RBBB & 88.88 & 99.75 & 99.41 \\
\hline Average & $\mathbf{9 0 . 8 4}$ & $\mathbf{9 6 . 0 3}$ & $\mathbf{9 5 . 9 8}$ \\
\hline
\end{tabular}

\section{DISCUSSION}

In order to prove that the performance of the proposed work is better, the results have been compared with some earlier works which were presented in the literature. Table 3 displays this comparison and from this table it can be noticed that EMD with AP, CD, and PCA is performing better as compared to other methods. The computational time for the proposed work is 3.323352 second.

Babaoğlu et al.used 18 features which was reduced by using PCA and classified it by using SVM to detect coronary artery diseases. Classification accuracy obtained by them was $79.17 \%$. The same SVM with BPSO and genetic algorithm (GA) was used by Babaoğlu et al. and they have achieved better result (81.46\%) as compared to SVM with PCA. Abdou et al. first decomposed and detected main events of the cardiac signal by using EMD. Then they have classified different types of arrhythmia by using PNN classifier and 67\% accuracy was obtained by them. Wavelet ECG features

Table 3. Comparison of the result obtained from the proposed work with other methods

\begin{tabular}{|l|l|l|l|}
\hline \multicolumn{1}{|c|}{ Reference } & \multicolumn{1}{c|}{ Features } & \multicolumn{1}{c|}{ Classifier } & \multicolumn{1}{c|}{ Accuracy (\%) } \\
\hline (Babaoğlu et al., 2010) & PCA & SVM & 79.17 \\
\hline (Babaoglu et al., 2010) & BPSO+GA & SVM & 81.46 \\
\hline (Abdou et al., 2018) & EMD & ANN & 67 \\
\hline (Gutiérrez-Gnecchi et al., 2017) & Wavelet & PNN & 92.75 \\
\hline (de Albuquerque et al., 2018) & Graph partitions & OPF, SVM & 91.22 \\
\hline Proposed work & EMD & RBFN & $\mathbf{9 5 . 9 8}$ \\
\hline
\end{tabular}


are classified with PNN classifier by Gutiérrez-Gnecchi et al. and they have obtained better result (91.75) as compared to SVM with different types of features. OPF and SVM classifier was used by de Albuquerque et al. for classification of different types of arrhythmia. They have classified five types of arrhythmias in their work and $91.22 \%$ classification accuracy was achieved by them.

\section{CONCLUSION}

An automatic computer-aided diagnosis system can identify the problem earlier before any critical stage. RBFN based automatic classification of four types of arrhythmia ECG signal is performing satisfactorily in this work. The EMD based signal decomposition technique is performing better as compared to other methods. An average sensitivity, specificity, and accuracy of $90.84 \%, 96.03 \%$, and 95.98\% are achieved respectively. The obtained classifiers performance result is compared with the previous works and it can be noticed that the proposed system is providing a better result and can be used in disease detection and diagnosis system. But this classifier performance will affect when the data set size will be large. At this stage, the number of hidden layers is required to increase. Further extension can be done for classifying different types of cardiac abnormalities by using the neural network with more numbers of hidden layers. Also, an optimized method is necessary to be used for better accuracy and is kept for future work. 


\section{REFERENCES}

Abdalla, F. Y., Wu, L., Ullah, H., Ren, G., Noor, A., \& Zhao, Y. (2019). ECG arrhythmia classification using artificial intelligence and nonlinear and nonstationary decomposition. Signal, Image and Video Processing, 13(7), 1-9. doi:10.1007/s11760-019-01479-4

Abdou, A.-D., Ngom, N. F., \& Niang, O. (2018). Classification and Prediction of Arrhythmias from Electrocardiograms Patterns Based on Empirical Mode Decomposition and Neural Network. Paper presented at the International Conference on e-Infrastructure and e-Services for Developing Countries.

Acharya, U. R., Sudarshan, V. K., Koh, J. E., Martis, R. J., Tan, J. H., Oh, S. L., \& Chua, K. P. et al. (2017). Application of higher-order spectra for the characterization of coronary artery disease using electrocardiogram signals. Biomedical Signal Processing and Control, 31, 31-43. doi:10.1016/j.bspc.2016.07.003

Afonso, V. X., Tompkins, W. J., Nguyen, T. Q., \& Luo, S. (1999). ECG beat detection using filter banks. IEEE Transactions on Biomedical Engineering, 46(2), 192-202. doi:10.1109/10.740882 PMID:9932341

Alonso-Atienza, F., Morgado, E., Fernandez-Martinez, L., García-Alberola, A., \& Rojo-Alvarez, J. L. (2013). Detection of life-threatening arrhythmias using feature selection and support vector machines. IEEE Transactions on Biomedical Engineering, 61(3), 832-840. doi:10.1109/TBME.2013.2290800 PMID:24239968

Babaoğlu, I., Findık, O., \& Bayrak, M. (2010). Effects of principle component analysis on assessment of coronary artery diseases using support vector machine. Expert Systems with Applications, 37(3), 2182-2185. doi:10.1016/j.eswa.2009.07.055

Babaoglu, İ., Findik, O., \& Ülker, E. (2010). A comparison of feature selection models utilizing binary particle swarm optimization and genetic algorithm in determining coronary artery disease using support vector machine. Expert Systems with Applications, 37(4), 3177-3183. doi:10.1016/j.eswa.2009.09.064

Blanco-Velasco, M., Weng, B., \& Barner, K. E. (2008). ECG signal denoising and baseline wander correction based on the empirical mode decomposition. Computers in Biology and Medicine, 38(1), 1-13. doi:10.1016/j. compbiomed.2007.06.003 PMID:17669389

Damerval, C., Meignen, S., \& Perrier, V. (2005). A fast algorithm for bidimensional EMD. IEEE Signal Processing Letters, 12(10), 701-704. doi:10.1109/LSP.2005.855548

Das, M. K., \& Ari, S. (2014). ECG beats classification using mixture of features. International Scholarly Research Notices, 2014, 2014. doi:10.1155/2014/178436 PMID:27350985

de Albuquerque, V. H. C., Nunes, T. M., Pereira, D. R., Luz, E. J. S., Menotti, D., Papa, J. P., \& Tavares, J. M. R. (2018). Robust automated cardiac arrhythmia detection in ECG beat signals. Neural Computing \& Applications, 29(3), 679-693. doi:10.1007/s00521-016-2472-8

Giri, D., Acharya, U. R., Martis, R. J., Sree, S. V., \& Lim, T.-C. (2013). Automated diagnosis of coronary artery disease affected patients using LDA, PCA, ICA and discrete wavelet transform. Knowledge-Based Systems, 37, 274-282. doi:10.1016/j.knosys.2012.08.011

Güler, İ., \& Übeylı, E. D. (2005). ECG beat classifier designed by combined neural network model. Pattern Recognition, 38(2), 199-208. doi:10.1016/j.patcog.2004.06.009

Gutiérrez-Gnecchi, J. A., Morfin-Magana, R., Lorias-Espinoza, D., del Carmen Tellez-Anguiano, A., ReyesArchundia, E., Méndez-Patiño, A., \& Castañeda-Miranda, R. (2017). DSP-based arrhythmia classification using wavelet transform and probabilistic neural network. Biomedical Signal Processing and Control, 32, 44-56. doi:10.1016/j.bspc.2016.10.005

Hagan, M. T., Demuth, H. B., \& Beale, M. (1997). Neural network design. PWS Publishing Co.

Hoekema, R., Uijen, G. J., \& Van Oosterom, A. (2001). Geometrical aspects of the interindividual variability of multilead ECG recordings. IEEE Transactions on Biomedical Engineering, 48(5), 551-559. doi:10.1109/10.918594 PMID:11341529

Hu, Y. H., Tompkins, W. J., Urrusti, J. L., \& Afonso, V. X. (1993). Applications of artificial neural networks for ECG signal detection and classification. Journal of Electrocardiology, 26, 66-73. PMID:8189150 
Huang, N. E., Shen, Z., Long, S. R., Wu, M. C., Shih, H. H., Zheng, Q., . . Liu, H. H. (1998). The empirical mode decomposition and the Hilbert spectrum for nonlinear and non-stationary time series analysis. Proceedings of the Royal Society of London. Series A: Mathematical, Physical and Engineering Sciences, 454(1971), 903995. doi:10.1098/rspa.1998.0193

Jiang, W., \& Kong, S. G. (2007). Block-based neural networks for personalized ECG signal classification. IEEE Transactions on Neural Networks, 18(6), 1750-1761. doi:10.1109/TNN.2007.900239 PMID:18051190

Kiranyaz, S., Ince, T., Pulkkinen, J., \& Gabbouj, M. (2011). Personalized long-term ECG classification: A systematic approach. Expert Systems with Applications, 38(4), 3220-3226. doi:10.1016/j.eswa.2010.09.010

Korürek, M., \& Doğan, B. (2010). ECG beat classification using particle swarm optimization and radial basis function neural network. Expert Systems with Applications, 37(12), 7563-7569. doi:10.1016/j.eswa.2010.04.087

Lewenstein, K. (2001). Radial basis function neural network approach for the diagnosis of coronary artery disease based on the standard electrocardiogram exercise test. Medical \& Biological Engineering \& Computing, 39(3), 362-367. doi:10.1007/BF02345292 PMID:11465892

Lin, C.-H. (2008). Frequency-domain features for ECG beat discrimination using grey relational analysis-based classifier. Computers \& Mathematics with Applications (Oxford, England), 55(4), 680-690. doi:10.1016/j. camwa.2007.04.035

Martis, R. J., Acharya, U. R., Prasad, H., Chua, C. K., Lim, C. M., \& Suri, J. S. (2013). Application of higher order statistics for atrial arrhythmia classification. Biomedical Signal Processing and Control, 8(6), 888-900. doi:10.1016/j.bspc.2013.08.008

Moody, G. B., \& Mark, R. G. (2001). The impact of the MIT-BIH arrhythmia database. IEEE Engineering in Medicine and Biology Magazine, 20(3), 45-50. doi:10.1109/51.932724 PMID:11446209

Moreira, J., de Leon Ferreira, A. C. P., \& Horváth, T. (2019). A General Introduction to Data Analytics. Wiley Online Library.

Palo, H. K., Mohanty, M. N., \& Chandra, M. (2016). Efficient feature combination techniques for emotional speech classification. International Journal of Speech Technology, 19(1), 135-150. doi:10.1007/s10772-016-9333-9

Phooi, S. K., \& Ang, L. (2006). Adaptive RBF neural network training algorithm for nonlinear and nonstationary signal. Paper presented at the 2006 International Conference on Computational Intelligence and Security. doi:10.1109/ICCIAS.2006.294170

Polat, K., \& Güneş, S. (2007). Detection of ECG Arrhythmia using a differential expert system approach based on principal component analysis and least square support vector machine. Applied Mathematics and Computation, 186(1), 898-906. doi:10.1016/j.amc.2006.08.020

Prasad, G. K., \& Sahambi, J. (2003). Classification of ECG arrhythmias using multi-resolution analysis and neural networks. Paper presented at the TENCON 2003. Conference on convergent technologies for Asia-Pacific region. doi:10.1109/TENCON.2003.1273320

Ram, R., \& Mohanty, M. N. (2019). Use of radial basis function network with discrete wavelet transform for speech enhancement. International Journal of Computational Vision and Robotics, 9(2), 207-223. doi:10.1504/ IJCVR.2019.098801

Sahani, M., \& Dash, P. K. (2018). Automatic power quality events recognition based on Hilbert Huang transform and weighted bidirectional extreme learning machine. IEEE Transactions on Industrial Informatics, 14(9), 3849-3858. doi:10.1109/TII.2018.2803042

Sufi, F., \& Khalil, I. (2010). Diagnosis of cardiovascular abnormalities from compressed ECG: A data miningbased approach. IEEE Transactions on Information Technology in Biomedicine, 15(1), 33-39. doi:10.1109/ TITB.2010.2094197 PMID:21097383

Tantawi, M. M., Revett, K., Salem, A.-B., \& Tolba, M. F. (2015). A wavelet feature extraction method for electrocardiogram (ECG)-based biometric recognition. Signal, Image and Video Processing, 9(6), 1271-1280. doi:10.1007/s11760-013-0568-5

Wang, G., Chen, X.-Y., Qiao, F.-L., Wu, Z., \& Huang, N. E. (2010). On intrinsic mode function. Advances in Adaptive Data Analysis, 2(03), 277-293. doi:10.1142/S1793536910000549 
Willems, J. L., \& Lesaffre, E. (1987). Comparison of multigroup logistic and linear discriminant ECG and VCG classification. Journal of Electrocardiology, 20(2), 83-92. doi:10.1016/S0022-0736(87)80096-1 PMID:2955068

Wu, Y., \& Rangayyan, R. M. (2009). An unbiased linear adaptive filter with normalized coefficients for the removal of noise in electrocardiographic signals. International Journal of Cognitive Informatics and Natural Intelligence, 3(4), 73-90. doi:10.4018/jcini.2009062305

Yu, S.-N., \& Chen, Y.-H. (2007). Electrocardiogram beat classification based on wavelet transformation and probabilistic neural network. Pattern Recognition Letters, 28(10), 1142-1150. doi:10.1016/j.patrec.2007.01.017

Saumendra Kumar Mohapatra is currently pursuing his Ph.D. degree at the department of computer science and engineering, Institute of Technical Education and Research, Siksha 'O' Anusandhan (Deemed to be University), Bhubaneswar, India. He has completed his master degree from Siksha 'O' Anusandhan (Deemed to be University) in 2017. His current research area includes ECG signal classification and analysis.

Mihir Narayan Mohanty is currently working as a Professor in the Department of Electronics and Communication Engineering, Institute of Technical Education and Research (FET), Siksha 'O' Anusandhan (Deemed to be University), Bhubaneswar, Odisha. He has received his M. Tech degree in Communication System Engineering from the Sambalpur University, Sambalpur, Odisha and obtained his PhD degree in Applied Signal Processing. He is the fellow of IE (I), and IETE. Also, he is the member of many professional societies including IEEE, IET etc. He has 25 years of teaching and research experience. He has published more than 300 papers in different Journals, Conferences including Book Chapters. He has authored two books. He is the successive reviewer of manuscripts from IEEE, Elsevier, Springer, IGI Global etc. His areas of research interests include Applied Signal and Image Processing, Wireless Communication, Antenna, Intelligent Signal Processing. 